\title{
Transcriptome profiling analysis reveals that CXCL2 is involved in anlotinib resistance in human lung cancer cells
}

\author{
Jun Lu', Wei Xu², Jie Qian', Shuyuan Wang1', Bo Zhang1', Lele Zhang ${ }^{1}$, Rong Qiao', Minjuan Hu', Yiming Zhao', \\ Xiaodong Zhao ${ }^{2^{*}}$ and Baohui Han ${ }^{1 *}$
}

From 11th International Multiconference "Bioinformatics of Genome Regulation and StructurelSystems Biology" - BGRSISB-

2018

Novosibirsk, Russia. 20-25 August 2018

\begin{abstract}
Background: Anlotinib has been demonstrated its anti-tumor efficacy on non-small cell lung cancer (NSCLC) in clinical trials at 3rd line. However, anlotinib resistance occurs during its administration, and the underlying mechanism is still unclear.

Methods: Anlotinib resistant lung cancer cell line NCl-H1975 was established in vitro. Toxicologic effects undergoing anlotinib stress were observed upon NCl-H1975 cells and anlotinib resistant NCl-H1975 cells, respectively. Transcriptome profiling was performed to screen anlotinib resistance-associated genes between NCl-H1975 cells and anlotinib resistant $\mathrm{NCl}-\mathrm{H} 1975$ cells. Functional assays were performed to examine the correlations between CXCL2 gene expression and anlotinib resistance.

Results: We found anlotinib inhibits cell proliferation and cell viability in NCl-1975 cells, whereas it attenuates these activities in anlotinib resistant NCl-H1975 cells. Transcriptome profiling analysis identified 769 anlotinib-responsive genes enriched in the biological processes of microtubule-based process, cytoskeleton organization, and wound healing. Furthermore, we found 127 genes are associated with anlotinib resistance. In particular, we demonstrated that CXCL2 contributes to anlotinib resistance in $\mathrm{NCl}-\mathrm{H} 1975$ cells.

Conclusions: This study suggested that CXCL2 is involved in anlotinib resistance in NCl-H1975 cells and provided an insight for understanding the resistant mechanism of anlotinib.
\end{abstract}

Keywords: Anlotinib, Drug resistance, CXCL2, Non-Small Cell Lung Cancer, Transcriptome

\section{Introduction}

Lung cancer is one of the most malignant cancers, and the non-small cell lung cancer (NSCLC) accounts for $80-85 \%$ of all lung cancer cases [1-3]. According to National Comprehensive Cancer Network (NCCN) guideline, the regimes of $1^{\text {st }}$ line and $2^{\text {nd }}$ line therapy bring great survival benefit for NSCLC patients, while the regimes of $3^{\text {rd }}$ line therapy have not been available so far

\footnotetext{
* Correspondence: xiaodongzhao@sjtu.edu.cn; 18930858216@163.com ${ }^{2}$ Shanghai Center for Systems Biomedicine, Shanghai Jiao Tong University, 800 Dong Chuan Rd, Shanghai 200240, China

'Department of Pulmonary Medicine, Shanghai Chest Hospital, Shanghai Jiao Tong University, 241 Huaihai West Rd, Shanghai 200030, China
}

$[4,5]$. Anlotinib is an oral multi-targeted tyrosine kinase receptor inhibitor (TKI) [6]. Previous studies demonstrated that anlotinib inhibits proliferation and induces apoptosis of tumor cells via selectively inhibiting VEGFR $(2 / 3)$, FGFR $(1-4)$, and PDGFR $(\alpha / \beta)[7,8]$. Clinical trials have indicated anlotinib prolongs progression free survival (PFS) and overall survival (OS) of NSCLC patients significantly at $3^{\text {rd }}$ line [9-11]. However, the median PFS of 5.37 months suggests anlotinib resistance occurred at later stage.

Investigating the mechanisms of anti-tumor drug resistance will improve the therapeutic efficiency or

(C) The Author(s). 2019 Open Access This article is distributed under the terms of the Creative Commons Attribution 4.0 International License (http://creativecommons.org/licenses/by/4.0/), which permits unrestricted use, distribution, and 
promote novel agent discovery. Previous studies have showed the NSCLC patients harboring EGFR positive mutations (L858R or 19 Del) acquired drug resistance with median PFS of about 10 months when they were treated with the first generation TKIs (Gefitinib, Erlotinib and Icotinib) [12-15]. More studies revealed that T790 M mutation and other gene over-expression account for the majority $[12,16,17]$. Based on these findings, scientists have found the $3^{\text {rd }}$ generation TKI AZD9291 is effectively for the patients harboring T790 $M$ mutation [18-20]. Investigating the resistant mechanism of anlotinib, a $3^{\text {rd }}$ line TKI, will be helpful to formulate the anlotinib-based therapeutic regime, and prolong PFS of NSCLC patients. In this study, we generated anlotinib resistant human lung cancer cell line NCI-H1975 in vitro, performed transcriptome analysis in NCI-H1975 cells and anlotinib resistant NCI-H1975 cells, and their anlotinib-treated counterpart with the aim to understand the mechanisms of anlotinib resistance.

\section{Materials and methods Cell culture}

Human NSCLC cell line NCI-H1975 was purchased from ATCC (the global bioresource center, https:// www.atcc.org/). TransDetect PCR Mycoplasma Detection Kit (TransGen, China) was used for excluding mycoplasma contamination. Cells were cultured in the mixed medium of 90\% RPMI 1640 (Gibco, USA), 10\% FBS (Gibco, USA), and $0.1 \mathrm{mg} / \mathrm{ml}$ streptomycin and 100 $\mathrm{U} / \mathrm{ml}$ penicillin. All cells were incubated at $37^{\circ} \mathrm{C}$ in a humidified incubator containing $5 \% \mathrm{CO}_{2}$.

\section{Generation of anlotinib resistant $\mathrm{NCl}-\mathrm{H} 1975$}

Anlotinib resistant NCI-H1975 was generated as previous reported [21, 22]. Briefly, $100 \mathrm{mg} / \mathrm{ml}$ ENU (sigma, USA) was exposed to totally $10^{7} \mathrm{NCI}-\mathrm{H} 1975$ cells for $24 \mathrm{~h}$. And then, gradient concentration of anlotinib treatment was performed to induce anlotinib resistant NCI-H1975 cells. At first five days, NCI-H1975 cells were exposed to anlotinib $(4 \mu \mathrm{g} / \mathrm{ml})$ and the medium was changed every day. Then anlotinib $(6 \mu \mathrm{g} / \mathrm{ml}, 8 \mu \mathrm{g} / \mathrm{ml}, 10 \mu \mathrm{g} / \mathrm{ml}$ and $12 \mu \mathrm{g} / \mathrm{ml})$ treatments were performed in the next two months. The last cells (about 100 cells) showed viability when exposing to anlotinib $(12 \mu \mathrm{g} / \mathrm{ml})$. Lastly, after about one month's proliferation, the anlotinib resistant NCI-H1975 cells were used to functional assays.

\section{Cell number and cell viability analysis}

For evaluating the effects of anlotinib on cell growth, $10^{5} \mathrm{NCI}-\mathrm{H} 1975$ cells were seeded in each well of 6-well plates, and then exposed to anlotinib $(8 \mu \mathrm{g} / \mathrm{ml})$ for $24 \mathrm{~h}$. Cell number changes in NCI-H1975 cells were observed with a phase-contrast microscope (Nikon, Japan). $10^{3}$ cells were seeded in 96-well plates and treated with anlotinib $(8 \mu \mathrm{g} / \mathrm{ml})$ for $24 \mathrm{~h}$. Cell viability was determined using Cell Counting Kit 8 (CCK8, Dojindo, Japan) by spectrophotometric plate reader (Omega Bio-Tek, USA).

\section{RNA-seq library construction}

RNA-seq library was performed as previous described [23-25]. Briefly, NCI-H1975 cells and anlotinib resistant NCI-H1975 cells were exposed to anlotinib $(8 \mu \mathrm{g} / \mathrm{ml})$ for $24 \mathrm{~h}$. Total RNA was extracted by Trizol (Life Technologies, USA) according to the manufacturer's protocol. mRNA was isolated from total RNA using Oligotex mRNA Mini Kit (Qiagen, Germany). Totally, $100 \mathrm{ng}$ mRNA was used for library construction. After cDNA reverse-transcription, end repair and ligation, the original library was amplified $10-12$ cycles in a thermal cycler using Q5 DNA Polymerase (NEB, USA). Lastly, the PCR products were performed by standard pair-end sequencing with $150 \mathrm{bp}$ reads with Illumina Next500 (Illumina, USA). Raw data are available in the EMBL database under accession number E-MTAB-5997 and E-MTAB-7068.

\section{RNA-seq data processing}

The sequencing quality of raw data was examined by FastQC software (Version 0.11.6). Qualified tags were mapped to reference genome (hg 38) by Tophat [26]. Cufflinks was used to characterize the differential transcription pattern [26]. Reads per kilo-base of transcript per million (RPKM) was used for detecting gene expression level. We performed differential gene expression analysis with RNA-seq data sets derived from NCI-H1975 and anlotinib-treated NCI-H1975, yielding 769 anlotinibresponsive genes. For the RNA-seq data sets generated with anlotinib resistant NCI-H1975 and anlotinib-treated anlotinib resistant NCI-H1975, we firstly removed genes whose transcription is modulated by anlotinib (RPKM in anlotinib resistant NCI-H1975/RPKM in anlotinibtreated anlotinib resistant NCI-H1975 > 1), the resulting genes were intersected with anlotinib responsive genes and the cancer gene pool (http://www.bushmanlab.org/ links/genelists), yielding 127 genes that are associated with Anlotinib resistance.

\section{Functional annotation and pathway analysis}

According to our previous studies [23, 25, 27], functional annotation and pathway analysis were performed by public bioinformatics resource platforms named Database for Annotation, Visualization and Integrated Discovery (DAVID) and Panther Classification System. Briefly, a list of 769 anlotinib-responsive genes was uploaded to DAVID bioinformatics resources 6.7 and performed Gene Ontology (GO) analysis and Kyoto Encyclopedia of Genes and Genomes (KEGG) pathway 
[28], and a list of 127 genes was uploaded to PANTHER Classification System and performed Panther Pathway analysis [29].

\section{Wound healing scratch assay}

$10^{5}$ NCI-H1975 cells were seeded on 6-well plates for $24 \mathrm{~h}$, and then were starved for $24 \mathrm{~h}$. Scratch wound was performed using $200 \mu \mathrm{L}$ pipet tip. Replace the medium with fresh 90\% RPMI 1640 medium (Gibco, USA) and $10 \%$ FBS (Gibco, USA). Meanwhile, other wells were treated with anlotinib $(4 \mu \mathrm{g} / \mathrm{ml})$, CXCL2 $(50 \mathrm{ng} / \mathrm{ml}$, Pepro Tech, USA), alone or together. Phase-contrast microscope (Nikon, Japan) was performed to capture images. The migration rate was calculated based on the change of wound width.

\section{Cell invasion assay}

Matrigel matrix (1:8 dilution, Corning, USA) was coated on transwell membrane. NCI-H1975 cells were seeded onto the top precoated chamber $\left(5 \times 10^{4}\right.$ cells per well for $24 \mathrm{~h}$ evaluation; $2 \times 10^{4}$ cells per well for $48 \mathrm{~h}$ evaluation) in $100 \mu \mathrm{L}$ of FBS-free medium containing anlotinib $(2 \mu \mathrm{g} / \mathrm{ml})$ and CXCL2 $(100 \mathrm{ng} / \mathrm{ml}$, Pepro Tech, USA), alone or together. The medium containing 15\% FBS (Gibco, USA) was placed in the bottom chamber. After $24 \mathrm{~h}$ or $48 \mathrm{~h}$ incubation, the invasive cells were fixed in $3.7 \%$ paraformaldehyde and stained with $0.1 \%$ crystal violet. Pictures were captured using fluorescence microscopy (Nikon, Japan). Cell numbers were examined by use of the microscopy affiliated software.

\section{Cell apoptosis assay}

Cell apoptosis detection was performed as previous described [27]. Annexin V-FITC/PI apoptosis kit (Zoman Biotechnology Co., Ltd., China) was used to examine the cell apoptosis. NCI-H1975 cells were exposed to CXCL2 (100 ng/ml, Pepro Tech, USA) and anlotinib $(4 \mu \mathrm{g} / \mathrm{ml})$, alone or together for $24 \mathrm{~h}$, and then stained with Annexin V-FITC and PI simultaneously. Flow cytometry (BD LSRFortessa, USA) was used for detecting apoptotic cells. PI-positive cells were designated end-stage apoptotic cells, and FITC-positive cells were designated early-stage apoptotic cells.

\section{Statistical analysis}

There are at least three biological replicates, excluding RNA-seq, for each sample. GraphPad Prism 5 was use for histogram and statistical analysis. Student's $t$-test was used to examine the raw data. Differences were considered significant at ${ }^{*} P<0.05,{ }^{* * *} P<0.01$ and ${ }^{* * * *} P<0.001$.

\section{Results}

Anlotinib-induced cytotoxicity attenuates in anlotinib resistant $\mathrm{NCl}-\mathrm{H} 1975$ cells

To understand the characteristics of anlotinib resistance, we firstly established anlotinib resistant
A

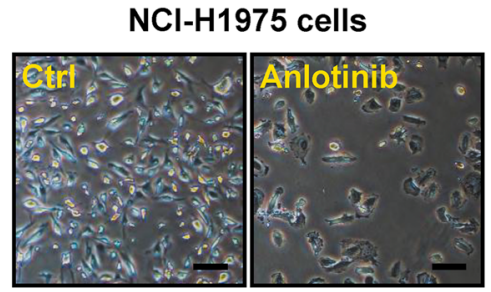

C

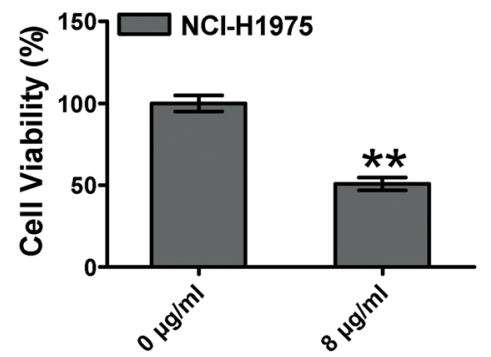

Anlotinib
B

Anlotinib Resistant $\mathrm{NCl}-\mathrm{H} 1975$ cells

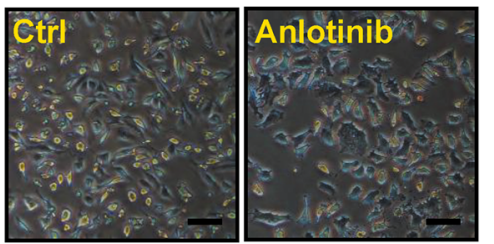

D

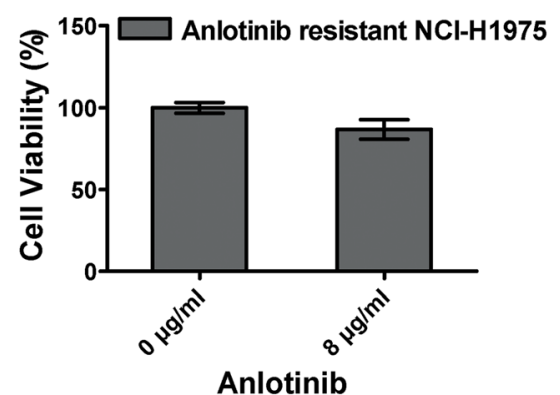

Fig. 1 Effect of anlotinib on cytotoxicity on NCl-H1975 cells and anlotinib resistant NCl-H1975 cells. a, b NCl-H1975 cells and anlotinib resistant $\mathrm{NCl}-\mathrm{H} 1975$ cells were treated with anlotinib $(8 \mu \mathrm{g} / \mathrm{ml})$ for $24 \mathrm{~h}$, and then the cell numbers were captured by phase-contrast microscopy. Scale bar, $50 \mu \mathrm{m}$. c, d Cell viabilities were examined when NCl-H1975 cells and anlotinib resistant NCl-H1975 cells were exposed to anlotinib $(8 \mu \mathrm{g} / \mathrm{ml})$ for $24 \mathrm{~h}$. Data are shown as the mean $\pm S D, n=3$, ${ }^{*} P<0.01$ 
NCI-H1975 cells in vitro. Under anlotinib stress, numbers of NCI-H1975 cells significantly decreased (Fig. 1a). However, numbers of anlotinib resistant NCI-H1975 cells were hardly affected, although exposing to same anlotinib stress as well (Fig. 1b). Furthermore, we examined the cell viability of these cells. After exposing to anlotinib for $24 \mathrm{~h}$, the cell viability of NCI-H1975 was decreased remarkably. In contrast, the cell viability of anlotinib resistant NCI-H1975 was hardly effected (Fig. 1c, d). These results indicated that anlotinib-induced cytotoxicity attenuates in anlotinib resistant NCI-H1975 cells.

\section{Identification of anlotinib-responsive genes in $\mathrm{NCl}-\mathrm{H} 1975$}

cells

We reasoned that the expression of genes potentially involved in anlotinib resistance should be modulated upon anlotinib treatment. Thus, we firstly performed transcriptome profiling analysis by RNA-seq both in NCI-H1975 and anlotinib-treated NCI-1975 cells. We removed the genes marginally expressed in NCI-H1975 (RPKM < 1) and then carried out differential expression analysis (expression fold $>2$ ). Compared with NCI-H1975, 769 genes were identified in anlotinib-treated NCI-H1975 (Fig. 2a, Additional file 1: Table S1). Heat map analysis suggested that $68 \%$ are down-regulated and $32 \%$ are up-regulated (Fig. $2 \mathrm{~b})$. Gene ontology (GO) analysis indicated that the 769 genes are significantly enriched in the biological processes of microtubule-based process, cytoskeleton organization, and wound healing (Fig. 2c). KEGG analysis suggested that these genes are enriched in the signaling pathways of steroid biosynthesis, gap junction, and TNF signaling pathway (Fig. 2d). Totally,

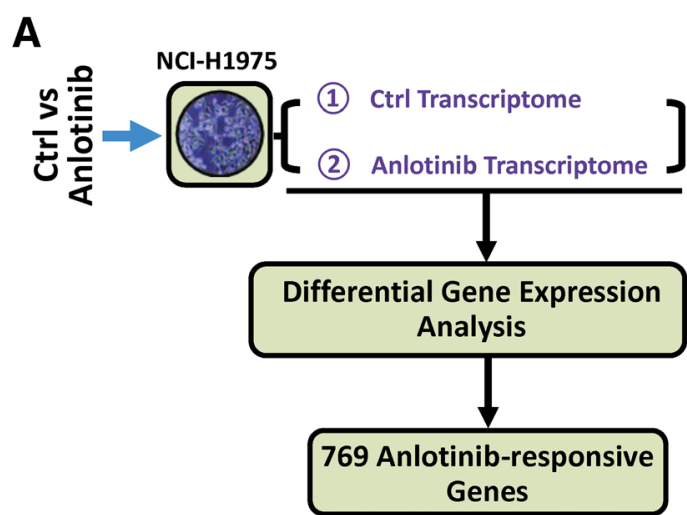

C

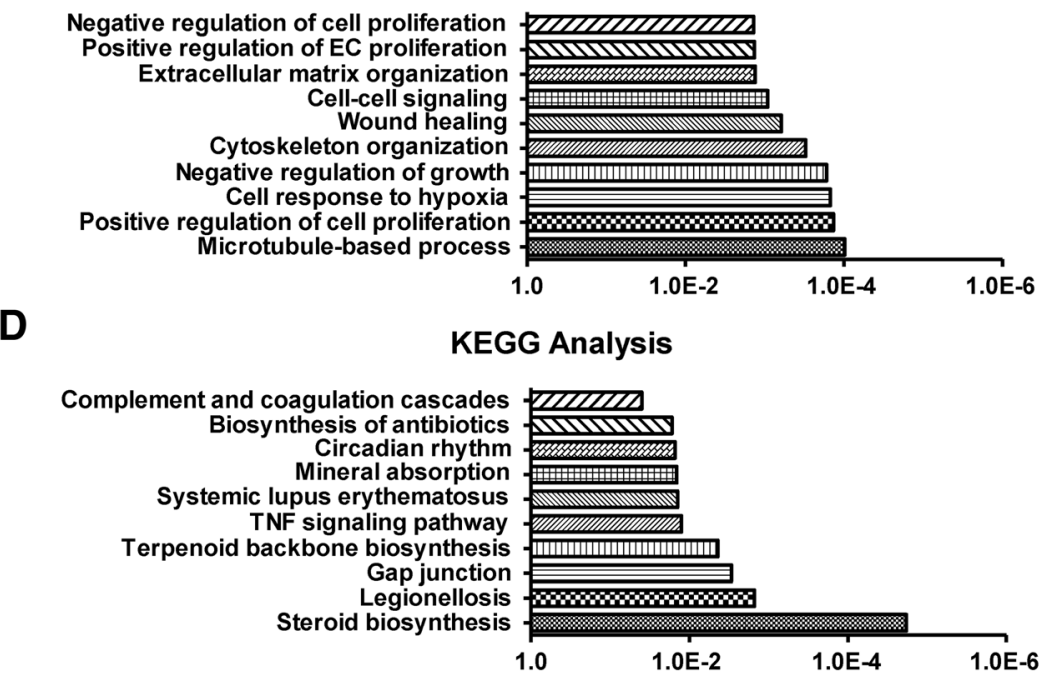

Go Analysis
B

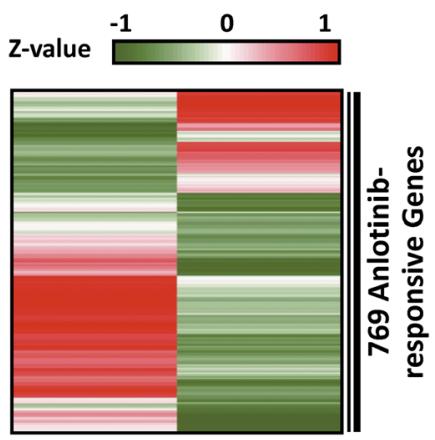

(1) 
these results suggest the set of 769 genes are anlotinib-responsive and involved in some cancer-related cellular activities.

\section{Identification of genes associated with anlotinib resistance in anlotinib resistant $\mathrm{NCl}-\mathrm{H} 1975$ cells}

To further understand the mechanisms of anlotinib resistance, we next performed RNA-seq analysis in anlotinib resistant NCI-H1975 cells and the anlotinib-treated counterpart. Among 769 anlotimib-responsive genes, we assumed the expression of genes that contribute to anlotinib resistance should be less modulated upon anlotinib treatment in anlotinib resistant cells (RPKM in anlotinib resistant NCI-H1975/ RPKM in anlotinib-treated anlotinib resistant NCI-H1975 < 1). This assumption allowed us to carry out differential gene expression analysis. Compared with anlotinib resistant NCI-H1975, 357 genes were identified in anlotinib-treated resistant NCI-H1975 cells with the criterion mentioned above. Furthermore, this set of 357 genes was compared with cancer gene pool (http://www.bushmanlab.org/links/genelists), yielding 127 genes (Fig. 3a, Additional file 2: Table S2). These genes are potentially involved in anlotinib resistance. As expected, the transcription levels of 127 genes decreased obviously in anlotinib-treated NCI-H1975 cells, while remaining less altered in anlotinib resistant NCI-H1975 and anlotinib-treated anlotinib resistant NCI-H1975 cells (Fig. 3b). Panther-Pathways analysis indicated that the 127 genes are involved in apoptosis signaling pathway, VEGF signaling pathway and angiogenesis (Fig. 3c).

CXCL2 is involved in the resistance in anlotinib resistant $\mathrm{NCl}-\mathrm{H} 1975$ cells

Previous studies have demonstrated that CXCL2 plays an important role in anti-tumor drug resistance on various types of cancer, including breast cancer, colorectal cancer and glioblastoma [30-32], and modulates the property of migration, invasion and apoptosis in cancer cells [33, 34]. Interestingly, CXCL2 is one of 127 candidate genes potentially associated with anlotinib resistance. In this study, we observed some cellular migration-related $\mathrm{GO}$ items (cytoskeleton organization, microtubule-based process, and wound healing) and

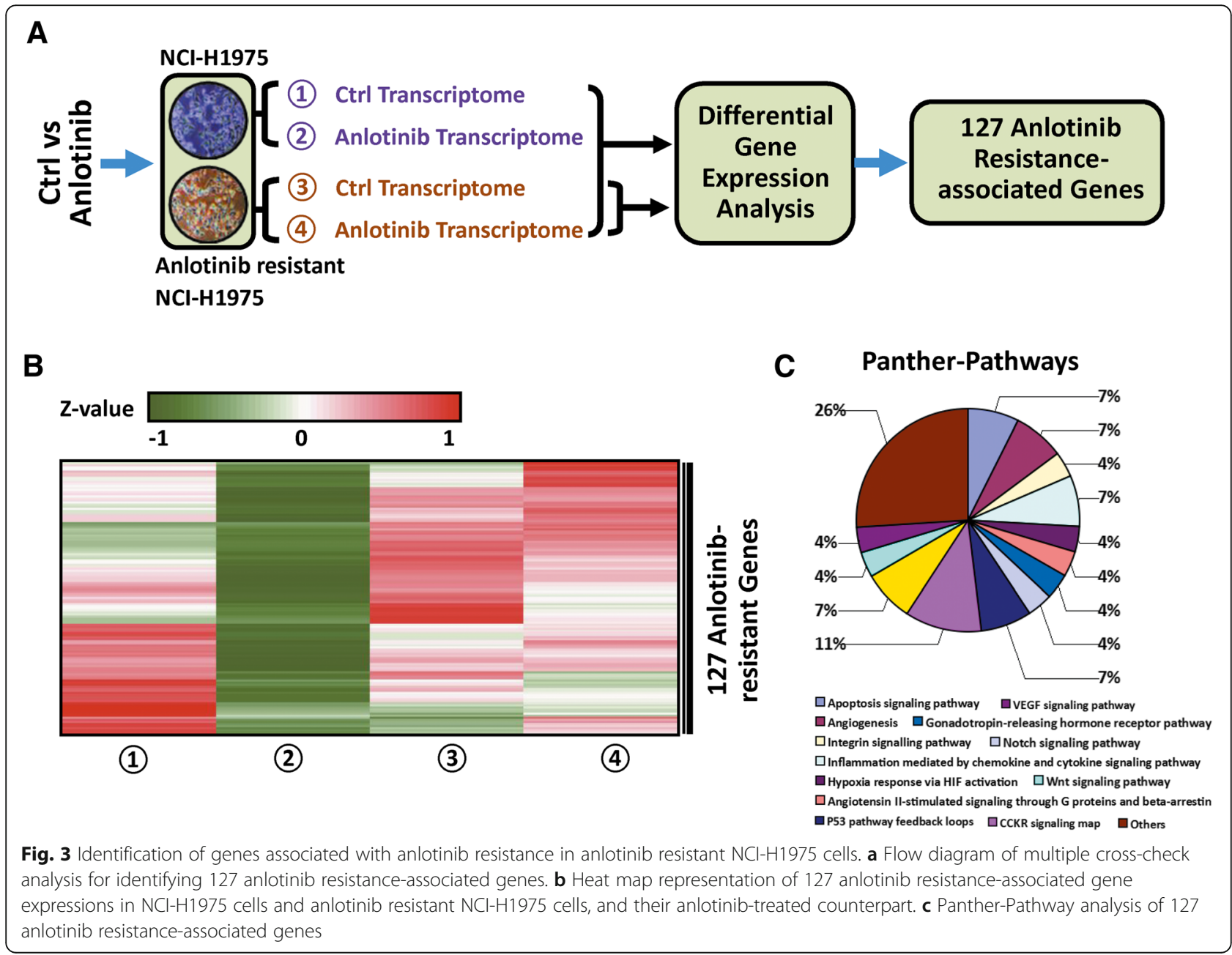


apoptosis signaling pathway are enriched when we investigated anlotinib resistance (Figs. 2c, 3c). We thus asked whether CXCL2 plays a role in the anlotinib resistance. To this end, we performed wound healing and transwell assays. We found exogenous CXCL2 obviously offsets anlotinib-induced NCI-H1975 cell migration inhibition (Fig. 4a, b). As shown in Fig. 4c and d, CXCL2 remarkably increases NCI-H1975 cell invasion, and promotes invasion of anlotinib-treated NCI-H1975 cells. Moreover, we found CXCL2 significant decreases

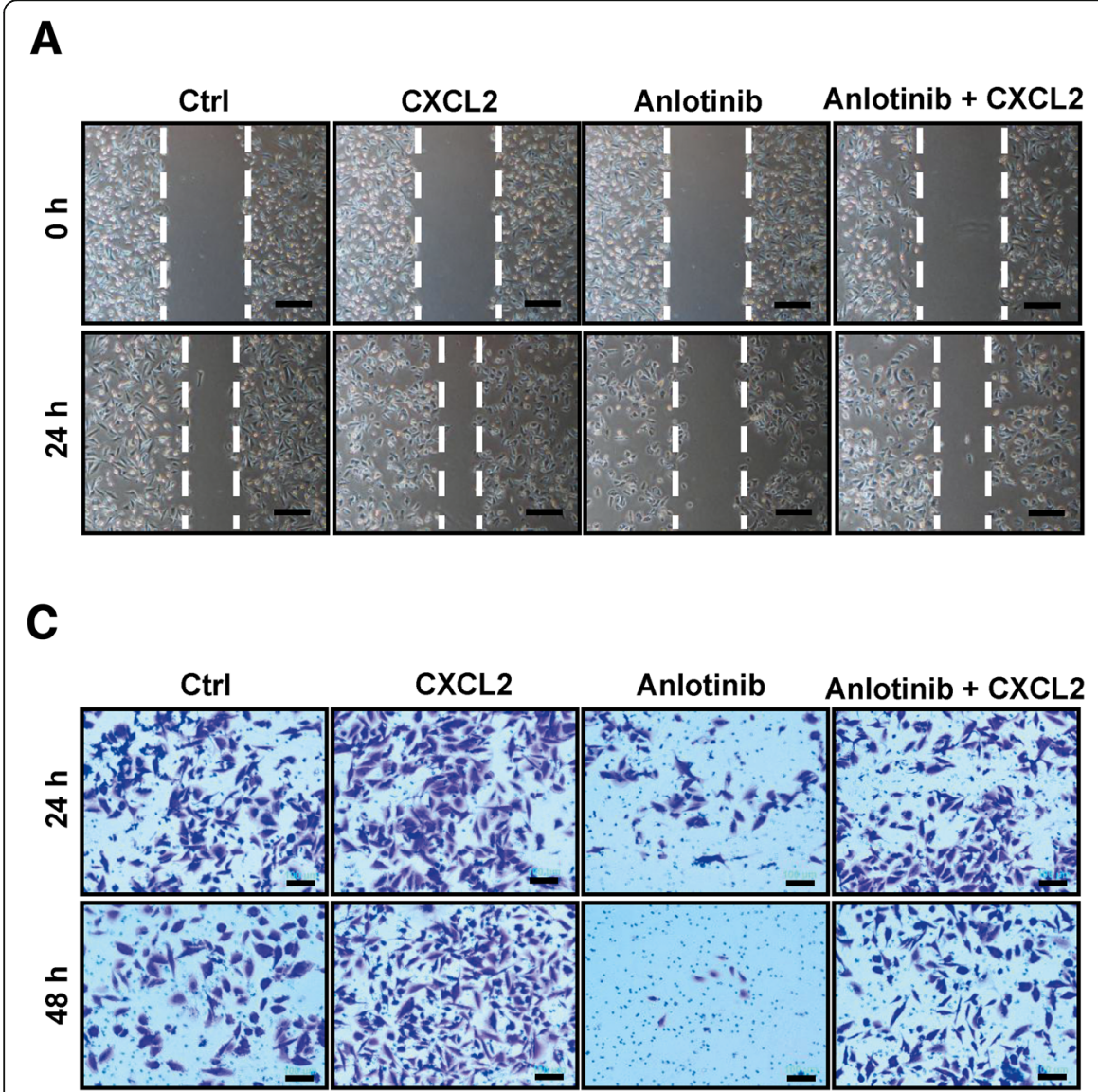

B

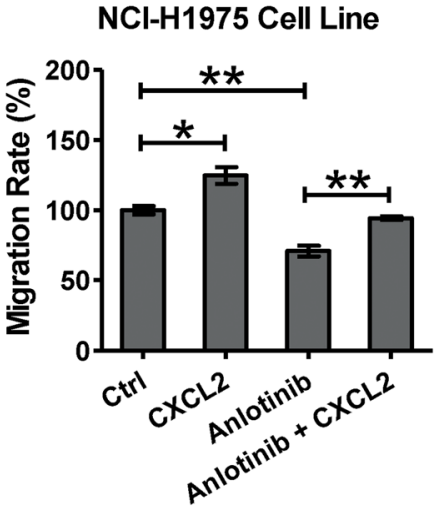

D

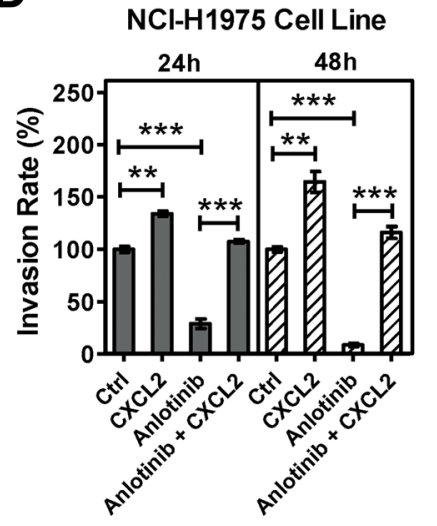

E

$\mathbf{F}$

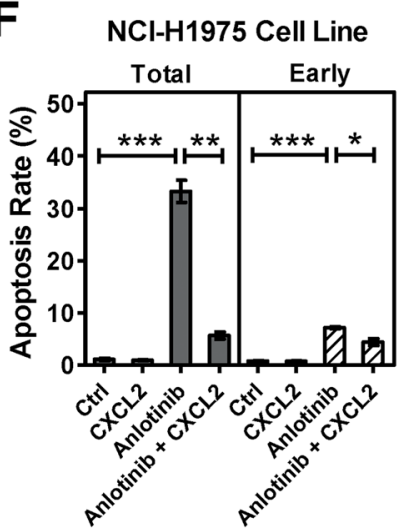

Fig. 4 CXCL2 is involved in anlotinib resistance in NCl-H1975 cells. a, b CXCL2 (50 ng/ml) and anlotinib $(4 \mu \mathrm{gg} / \mathrm{ml})$ were performed on NCl-H1975 cells, alone or together for $24 \mathrm{~h}$. Migration rate was examined by wound healing scratch assay. Bars $=$ mean $\pm \mathrm{SD}, n=3,{ }^{*} P<0.05,{ }^{*} P<0.01$. Scale bar, $100 \mu \mathrm{m}$ c, d CXCL2 $(100 \mathrm{ng} / \mathrm{ml})$ and anlotinib $(2 \mu \mathrm{g} / \mathrm{ml})$ were performed on NCl-H1975 cells, alone or together, for $24 \mathrm{~h}$. Invasion rate was analyzed based on transwell assays. Bars $=$ mean $\pm \mathrm{SD}, n=3,{ }^{* *} P<0.01,{ }^{* * *} P<0.001$. Scale bar, $100 \mu \mathrm{m}(\mathrm{E}, \mathrm{F}) \mathrm{NCl}-\mathrm{H} 1975$ cells were exposed to CXCL2 $(100 \mathrm{ng} / \mathrm{ml})$ and anlotinib $(4 \mathrm{\mu g} / \mathrm{ml})$, alone or together for $24 \mathrm{~h}$. Ratio of total apoptosis and early apoptosis were examined based on flow cytometric detection. Data are shown as mean $\pm \mathrm{SD}, n=3,{ }^{*} P<0.05,{ }^{* *} P<0.01,{ }^{* * *} P<0.001$ 
anlotinib-induced apoptosis (both total apoptosis and early apoptosis) in NCI-H1975 cells (Fig. 4e, f). Overall, these results indicated CXCL2 is involved in the resistance in anlotinib resistant NCI-H1975 cells.

\section{Discussion}

Our previous studies have shown that anlotinib is an effective anti-tumor agent for NSCLC at $3^{\text {rd }}$ line [9-11, 35]. It is also demonstrated that anlotinib selectively inhibits VEGFR (2/3), FGFR (1-4), and PDGFR $(\alpha / \beta)$ [7, 8]. However, drug resistance is inevitable in the last stage of cancer therapy.

NSCLC patients harboring EGFR (L858R and 19 Del) mutation received great benefit in more than 10 years ago, due to the finding of Gefitinib [36]. Although the first generation TKIs (Gefitinib, Erlotinib and Icotinib) have demonstrated its efficacy upon those NSCLC patients, acquired resistance will occurs after a median about 10 months therapy [12-15]. Subsequently, the scientists focused on the resistant mechanism study, and found that T790 M mutation, MET amplification, HER-2 mutation, and other gene alteration are contributed to acquired resistance [12, 16, 17]. Because of the patients harboring T790 M accounts for about 50\% of the acquired resistance, scientists next screened out AZD9291, and brought greatly benefit for those patients with T790 M mutation [18-20].

In recent years, the high-throughput sequencing technology has served as an important platform to characterize the acquired resistance [37]. Understanding the underlying anlotinib resistant mechanism will benefit the therapeutic outcome. To address this issue, in the present study we performed transcriptome profiling analysis in NCI-H1975 cells, anlotinib resistant NCI-H1975 cells, and their anlotinib-treated cells. We identified a set of 127 genes that are potentially associated with anlotinib resistance via transcriptome profiling analysis (Fig. 3b).

Among these 127 candidate genes CXCL2 raised our attention. CXCL2 is an important cytokine, which usually is involved in wound healing, cancer metastasis, apoptosis, and angiogenesis [33, 34, 38]. Recent studies revealed CXCL2 is also associated with acquired resistance in breast cancer, colorectal cancer and glioblastoma [30-32]. These phenomena gave us a hint that whether CXCL2 plays an important role in anlotinib resistance. To address this issue, we performed function assays to examine the relevance between CXCL2 and anlotinib resistance. As we expected, our results showed that anlotinib-induced apoptosis and the inhibition of migration and invasion in NCI-H1975 cells were significantly recovered when supplementing exogenous CXCL2, suggesting an important link between CXCL2 and anlotinib resistance.

\section{Conclusions}

In brief, our findings revealed a novel resistant mechanism of anlotinib and provided a basis for circumventing anlotinib resistance.

\section{Additional files}

Additional file 1: This file includes the mRNA levels of anlotinibresponsive 769 genes in $\mathrm{NCl}-\mathrm{H} 1975$ cells, anlotinib resistant $\mathrm{NCl}-\mathrm{H} 1975$ cells, and their anlotinib-treated cells. (XLSX $55 \mathrm{~kb}$ )

Additional file 2: This file includes the mRNA levels of anlotinib resistance-related 127 genes in $\mathrm{NCl}-\mathrm{H} 1975$ cells, anlotinib resistant $\mathrm{NCl}$ H1975 cells, and their anlotinib-treated cells. (XLSX 16 kb)

\section{Abbreviations}

CXCL2: C-X-C Motif Chemokine Ligand 2; DAVID: Database for Annotation, Visualization and Integrated Discovery; EGFR: Epidermal Growth Factor Receptor; ENU: N-ethyl-N-nitrosourea; GO: Gene Ontology; KEGG: Kyoto Encyclopedia of Genes and Genomes; NCCN: National Comprehensive Cancer Network; NSCLC: Non-Small Cell Lung Cancer; OS: Overall Survival; PDGFR: Platelet-derived Growth Factor Receptor; FGFRFibroblast Growth Factor Receptor; PFS: Progression Free Survival; RPKM: Reads per Kilo-base of Transcript per Million; TKI: Tyrosine Kinase Receptor Inhibitor; VEGFR: Vascular Endothelial Growth Factor Receptor 2

\section{Acknowledgements}

The authors would like to thank Dr. Jun Wu for assistance of bioinformatic analysis and analysis, thank Dr. Xunqiang Wang for providing anlotinib.

\section{Funding}

Publication of this article was supported by the program of system biomedicine innovation center from Shanghai Jiao Tong University (Project No. 15ZH4009); the key program of translational medicine from Shanghai Jiao Tong University School of Medicine (Project No. 15ZH1008); National Natural Science Foundation of China (Project No. 81673015); and the project of Science and Technology Commission of Shanghai Municipality (Project No. 16140902700).

\section{Availability of data and materials}

The profiling datasets of this article are available in the EMBL database under accession number E-MTAB-5997 and E-MTAB-7068. Other data including anlotinib resistant $\mathrm{NCl}-\mathrm{H} 1975$ cell line are available based on reasonable request.

\section{About this supplement}

This article has been published as part of BMC Medical Genomics Volume 12 Supplement 2, 2019: Selected articles from BGRSISB-2018: medical genomics. The full contents of the supplement are available online at https:// bmcmedgenomics.biomedcentral.com/articles/supplements/volume-12supplement-2.

\section{Authors' contributions}

Experiments were conceived and designed by $\mathrm{BH}, \mathrm{XZ}$ and JL. Cell assays were performed by $J$, JQ, BZ, SW, RQ, MH and $Y Z$. RNA-seq library was performed by $W X$ and LZ. Bioinformatics analysis and statistical analysis were performed by $L Z$ and $J L$. The manuscript was written by $J$, and revised by $\mathrm{XZ}$ and $\mathrm{BH}$. All of the authors have read and approved the final manuscript.

Ethics approval and consent to participate

Not applicable.

Consent for publication

Not applicable.

Competing interests

The authors declare that they have no competing interests. 


\section{Publisher's Note}

Springer Nature remains neutral with regard to jurisdictional claims in published maps and institutional affiliations.

Published: 13 March 2019

\section{References}

1. Jemal A, Bray F, Center MM, Ferlay J, Ward E, Forman D. Global cancer statistics. CA Cancer J Clin. 2011;61(2):69-90.

2. Siegel RL, Miller KD, Jemal A. Cancer statistics, 2018. 2018;68(1):7-30.

3. Lu J, Han BH. Liquid biopsy promotes non-small cell Lung Cancer precision therapy. Technol Cancer Res T. 2018;7(17):1-7.

4. Ettinger DS, Wood DE, Aisner DL, Akerley W, Bauman J, Chirieac LR, et al. Non-small cell Lung Cancer, version 5.2017, NCCN clinical practice guidelines in oncology. J Natl Compr Canc Net. 2017;15(4):504-35.

5. Ettinger DS, Wood DE, Akerley W, Bazhenova LA, Borghaei H, Camidge DR, et al. NCCN guidelines insights: non-small cell Lung Cancer, version 4.2016 J Natl Compr Canc Net. 2016;14(3):255-64.

6. Sun Y, Niu W, Du F, Du C, Li S, Wang J, et al. Safety, pharmacokinetics, and antitumor properties of anlotinib, an oral multi-target tyrosine kinase inhibitor, in patients with advanced refractory solid tumors. J Hematol Oncol. 2016;9(1):105.

7. Lin B, Song $X$, Yang D, Bai D, Yao Y, Lu N. Anlotinib inhibits angiogenesis via suppressing the activation of VEGFR2, PDGFRbeta and FGFR1. Gene. 2018; 654:77-86.

8. Xie C, Wan X, Quan H, Zheng M, Fu L, Li Y, Lou L. Preclinical characterization of anlotinib, a highly potent and selective vascular endothelial growth factor receptor-2 inhibitor. Cancer Sci. 2018;109(4):120719.

9. Han BH, Li K, Zhao YZ, Li BL, Cheng Y, Zhou JY, et al. Anlotinib as a thirdline therapy in patients with refractory advanced non-small-cell lung cancer: a multicentre, randomised phase II trial (ALTER0302). Br J Cancer. 2018. https://doi.org/10.1038/bjc.2017.478

10. Han BH, Li K, Wang QM, Zhao YZ, Zhang L, Shi JH, et al. Efficacy and safety of third-line treatment with anlotinib in patients with refractory advanced non-small-cell lung cancer (ALTER-0303): a randomised, double-blind, placebo-controlled phase 3 study. Lancet Oncol. 2017;18:S3.

11. Han BH, Li K, Wang QM, Zhang L, Shi JH, Wang ZH, Cheng Y, et al. Effect of Anlotinib as a third-line or further treatment on overall survival of patients with advanced non-small cell Lung Cancer: The ALTER 0303 Phase 3 Randomized Clinical Trial. JAMA Oncol. 2018. https://doi.org/10.1001/ jamaoncol.2018.3039.

12. Camidge DR, Pao W, Sequist LV. Acquired resistance to TKIs in solid tumours: learning from lung cancer. Nat Rev Clin Oncol. 2014;11(8):473-81.

13. Gerber NK, Yamada Y, Rimner A, Shi WJ, Riely GJ, Beal K, et al. Erlotinib versus radiation therapy for brain metastases in patients with EGFR-mutant Lung adenocarcinoma. Int J Radiat Oncol. 2014:89(2):322-9.

14. Mok TS, Wu YL, Thongprasert S, Yang CH, Chu DT, Saijo N, Sunpaweravong $\mathrm{P}$, et al. Gefitinib or carboplatin-paclitaxel in pulmonary adenocarcinoma. N Engl J Med. 2009;361(10):947-57.

15. Shih $J Y$, Gow CH, Yang PC. EGFR mutation conferring primary resistance to gefitinib in non-small-cell lung cancer. N Engl J Med. 2005:353(2):207-8.

16. Jakobsen KR, Demuth C, Madsen AT, Hussmann D, Vad-Nielsen J, Nielsen AL, et al. MET amplification and epithelial-to-mesenchymal transition exist as parallel resistance mechanisms in erlotinib-resistant, EGFR-mutated, NSCLC HCC827 cells. Oncogenesis. 2017:6(4):e307.

17. Joseph NA, Chiou SH, Lung Z, Yang CL, Lin TY, Chang HW, et al. The role of HGF-MET pathway and CCDC66 cirRNA expression in EGFR resistance and epithelial-to-mesenchymal transition of lung adenocarcinoma cells. J Hematol Oncol. 2018;11(1):74.

18. Parra HJS, Noto L, Galetta D, Ferrau F, Gebbia V, Tassone P, et al. A phase II, noncomparative, open label, multicentre, study of AZD9291 in patients with locally advanced or metastatic EGFR mutated "T790M undetectable or unknown" non-small cell lung cancer (stage IIB-IV) after no immediate prior EGFR TKI (OSIRIS study). J Clin Oncol. 2017;35(15_suppl):TPS9107.

19. Ku BM, Bae YH, Koh J, Sun JM, Lee SH, Ahn JS, et al. AZD9291 overcomes T790 M-mediated resistance through degradation of EGFR (L858R/T790M) in non-small cell lung cancer cells. Invest New Drug. 2016;34(4):407-15.

20. Vallee A, Audigier-Valette C, Herbreteau G, Merrien J, Tessonnier L, Theoleyre $S$, et al. Rapid clearance of circulating tumor DNA during treatment with AZD9291 of a lung cancer patient presenting the resistance EGFR T790M mutation. Lung Cancer. 2016:91:73-4.

21. Engelman JA, Zejnullahu K, Mitsudomi T, Song YC, Hyland C, Park JO, et al. MET amplification leads to gefitinib resistance in lung cancer by activating ERBB3 signaling. Science. 2007;316(5827):1039-43.

22. Uchibori K, Inase N, Nishio M, Fujita N, Katayama R. Identification of mutation accumulation as resistance mechanism emerging in first-line Osimertinib treatment. J Thorac Oncol. 2018;13(7):915-25.

23. Lu J, Chen J, Xu N, Wu J, Kang Y, Shen T, Kong H, et al. Activation of AlFM2 enhances apoptosis of human lung cancer cells undergoing toxicological stress. Toxicol Lett. 2016;258:227-36.

24. Zhang XL, Wu J, Wang J, Shen T, Li H, Lu J, et al. Integrative epigenomic analysis reveals unique epigenetic signatures involved in unipotency of mouse female germline stem cells. Genome Biol. 2016;17(1):162.

25. Lu J, Chen J, Kang Y, Wu J, Shi H, Fu Y, et al. Jinfukang induces cellular apoptosis through activation of Fas and DR4 in A549 cells. Oncol Lett. 2018. https://doi.org/10.3892/ol.2018.9149.

26. Trapnell C, Roberts A, Goff L, Pertea G, Kim D, Kelley DR, et al. Differential gene and transcript expression analysis of RNA-seq experiments with TopHat and cufflinks. Nat Protoc. 2012;7(3):562-78.

27. Lu J, Zhang $X$, Shen $T, M a C$, Wu J, Kong $H$, et al. Epigenetic profiling of H3K4Me3 reveals herbal medicine Jinfukang-induced epigenetic alteration is involved in anti-Lung Cancer activity. Evid Based Complement Alternat Med. 2016. https://doi.org/10.1155/2016/7276161.

28. Dennis G Jr, Sherman BT, Hosack DA, Yang J, Gao W, Lane HC, et al. DAVID: database for annotation, visualization, and integrated discovery. Genome Biol. 2003;4(5):P3.

29. Mi HY, Muruganujan A, Casagrande JT, Thomas PD. Large-scale gene function analysis with the PANTHER classification system. Nat Protoc. 2013; 8(8):1551-66.

30. Han J, Jun Y, Kim SH, Hoang HH, Jung Y, Kim S, et al. Rapid emergence and mechanisms of resistance by U87 glioblastoma cells to doxorubicin in an in vitro tumor microfluidic ecology. Proc Natl Acad Sci U S A. 2016;113(50):14283-8.

31. Jia DY, Li L, Andrew S, Allan D, Li XG, Lee J, et al. An autocrine inflammatory forward-feedback loop after chemotherapy withdrawal facilitates the repopulation of drug-resistant breast cancer cells. Cell Death Dis. 2017;8(7):e2932.

32. De Porras VR, Bystrup S, Martínez-Cardús A, Pluvinet R, Sumoy L, Howells L, et al. Curcumin mediates oxaliplatin-acquired resistance reversion in colorectal cancer cell lines through modulation of CXC-chemokine/NF-KB signalling pathway. Sci Rep. 2016;6:24675.

33. Song $X F$, Wang $Z$, Jin $Y$, Wang $Y$, Duan WB. Loss of miR-532-5p in vitro promotes cell proliferation and metastasis by influencing CXCL2 expression in HCC. Am J Transl Res. 2015;7(11):2254-61.

34. Gao Y, Guan ZF, Chen JQ, Xie HJ, Yang Z, Fan JH, et al. CXCL5/CXCR2 axis promotes bladder cancer cell migration and invasion by activating PI3K AKT-induced upregulation of MMP2/MMP9. Int J Oncol. 2015:47(2):690-700.

35. Lu J, Zhong H, Chu T, Zhang XY, Li R, Sun JY, et al. Role of anlotinibinduced CCL2 decrease in antiangiogenesis and response prediction for non-small cell lung cancer therapy. Eur Respir J. 2018. https://doi.org/10. 1183/13993003.01562-2018

36. Paez JG, Janne PA, Lee JC, Tracy S, Greulich H, Gabriel S, et al. EGFR mutations in lung cancer: correlation with clinical response to gefitinib therapy. Science. 2004;304(5676):1497-500.

37. Collisson EA, Campbell JD, Brooks AN, Berger AH, Lee W, Chmielecki J, et al. Comprehensive molecular profiling of lung adenocarcinoma. Nature. 2014; 511(7511):543-50

38. Al-Alwan LA, Chang Y, Mogas A, Halayko AJ, Baglole CJ, Martin JG, et al. Differential roles of CXCL2 and CXCL3 and their receptors in regulating normal and asthmatic airway smooth muscle cell migration. J Immunol. 2013;191(5):2731-41. 